\title{
Orthogonalized Partial Directed Coherence for Functional Connectivity Analysis of Newborn EEG
}

\author{
Amir H. Omidvarnia ${ }^{1, *}$, Ghasem Azemi ${ }^{1,2}$, Boualem Boashash ${ }^{1,3}$, John M. O' Toole ${ }^{4}$, \\ Paul Colditz ${ }^{1}$, and Sampsa Vanhatalo ${ }^{1,5}$ \\ ${ }^{1}$ Centre for Clinical research, The University of Queensland, Brisbane, Australia \\ \{a.omidvarnia, g.azemi,p.colditz\}@uq.edu.au \\ ${ }^{2}$ Department of Electrical Engineering, Razi University, Kermanshash, Iran \\ ${ }^{3}$ Qatar University College of Engineering, Doha, Qatar \\ boualem@qu. edu.qa \\ ${ }^{4}$ DeustoTech, University of Deusto, Bilbao, Spain \\ j.otooledieee.org \\ ${ }^{5}$ Department of Clinical Neurophysiology, Helsinki University Hospital, Helsinki, Finland \\ Sampsa.vanhatalo@helsinki.fi
}

\begin{abstract}
The aim of this study is to develop a time-frequency method and test its applicability to investigating directional cortical connectivity in the newborn brain considering the effect of volume conduction. We modified time-varying partial directed coherence (tv-PDC) based on orthogonalization of the MVAR model coefficients to deal with the effect of mutual independent sources. The novel measure was then tested using a simulated signal with feature dimensions relevant to EEG activity. From the neonatal EEG responses evoked by flash light stimuli $(1 \mathrm{~Hz})$, we extracted the directional interactions over time within each hemisphere. The results suggest that the method is able to detect directed information flow within a sub-second time scale in nonstationary multichannel signals (such as newborn EEG) and attenuate the problematic effect of volume conduction for multichannel EEG connectivity analysis.
\end{abstract}

\section{Introduction}

The human brain performs its sensory and cognitive functions by dynamically employing highly complex and interlaced neuronal networks. Better understanding of these network functions may open insights to pathophysiological mechanisms of neurological development and disease [1]. Due to its non-invasive nature, high temporal resolution and low cost, brain connectivity analysis using scalp EEG has received attention in recent years $[2,3]$.

Several methods have been developed for assessing directed interactions from neurological signals (reviewed in [4]). Among these, Multivariate autoregressive (MVAR) model-based methods have been widely used for neurophysiological signal analysis [2, 5, 6]. An MVAR process is able to model interactions between EEG channels in the form of linear difference equations and allows the direction of information flow between channels including direct and indirect influences [7]. Granger causality index [8], Directed Coherence [9], Partial Directed Coherence (PDC) [9], 
Directed Transfer Function (DTF) [10] and direct Directed Transfer Function (dDTF) [11] are MVAR-based measures that have been introduced to determine directional influence in multivariate systems. They have also been validated using simulated models $[7,9,12]$. The original versions of these measures assume that the underlying signals are stationary and their interactions are constant over time. However, none of these assumptions is satisfied for EEG signals which represent a non-stationary behavior and are generated through the time-varying complex system of the brain. Therefore, time-varying MVAR-based connectivity measures have been proposed to account for the non-stationary nature of the EEG signals.

A serious challenge in scalp EEG/MEG connectivity analysis is associated with the effect of volume conduction where the activity of a single source is reflected in many channels [13]. It may lead to the illusive connectivity between close-by electrodes, while they are not linked by factual brain interactions necessarily [13]. It becomes even more problematic for the MVAR-based measures, because the fitted MVAR coefficients on the multichannel EEG/MEG data might be considerably affected by volume conduction effects ([14], page 80). A potential solution is to perform the EEG/MEG connectivity analysis at the source level. It, however, needs a separate processing module for EEG/MEG source localization and solving the inverse problem. A recent EEG correlation analysis study [15] suggests the use of orthogonalized signals instead of the original ones to discount the effect of volume conduction. In this paper, we adapt the idea for MVAR-based EEG connectivity analysis and develop a novel extension of the classical PDC (called orthogonalized PDC or OPDC) to minimize the effect of mutual sources in multivariate signals. Then, we test it with a simulated model as well as newborn EEG traces evoked by flash light stimuli. By extracting time-frequency (T-F) connectivity maps and using a statistical testing approach, statistically significant $\mathrm{T}$-F elements of the connectivity maps are determined.

\section{Materials and Methods}

\subsection{Time-Varying PDC Measure}

For a time series $\boldsymbol{y}(n) \in \mathbb{R}^{M}$ with length $L$, a time-varying MVAR model of order $p$ is defined as:

$$
\left[\begin{array}{c}
y_{1}(n) \\
\vdots \\
y_{N}(n)
\end{array}\right]=\sum_{r=1}^{p} \boldsymbol{A}_{r}(n)\left[\begin{array}{c}
y_{1}(n-r) \\
\vdots \\
y_{M}(n-r)
\end{array}\right]+\left[\begin{array}{c}
w_{1}(n) \\
\vdots \\
w_{M}(n)
\end{array}\right]
$$

where $\left[w_{1} \cdots w_{M}\right]^{T}=\boldsymbol{w}$ is a normally distributed white noise vector and $M$ denotes the number of channels. The matrices $\mathbf{A}_{\mathrm{r}}$ are given by:

$$
\boldsymbol{A}_{\boldsymbol{r}}(n)=\left[\begin{array}{ccc}
a_{11}^{r}(n) & \cdots & a_{1 M}^{r}(n) \\
\vdots & \ddots & \vdots \\
a_{M 1}^{r}(n) & \cdots & a_{M M}^{r}(n)
\end{array}\right]
$$

for $r=1, \ldots, p$. The parameter $a_{k l}^{r}(n)$ reflects the time-varying linear relationship between channel $k$ and channel $l$ at the delay $r$.

The PDC measure is based on the concept of Granger causality [9]. A dynamical process $\mathrm{X}$ is said to Granger-cause a dynamical process $\mathrm{Y}$, if the prediction of the 
process $\mathrm{Y}$ is enhanced using the information of the past of process $\mathrm{X}$ compared to the knowledge of the past of process $Y$ alone [12]. Such relationship between different channels of a multivariate signal is reflected to the MVAR parameters $\boldsymbol{A}_{\boldsymbol{r}}(n)$ in the time (Eq. (1)) and frequency domains (Eq. (3)).

$$
\boldsymbol{A}(n, f)=I-\left.\sum_{r=1}^{p} \boldsymbol{A}_{r}(n) z^{-r}\right|_{z=e^{j 2 \pi f}}
$$

$f \quad$ varies within the range of 0 to the Nyquist rate. In this paper, $\boldsymbol{A}_{r}(n)$ are estimated using dual extended Kalman filter (DEKF) [7]. The time-varying version of the PDC is then defined as [7]:

$$
\pi_{k l}(n, f) \triangleq \frac{\left|A_{k l}(n, f)\right|}{\sqrt{\boldsymbol{a}_{l}^{H}(n, f) \boldsymbol{a}_{l}(n, f)}}
$$

where $\boldsymbol{a}_{l}(n, f)$ is the $l$ 'th column, $A_{k l}(n, f)$ is the $k l$ 'th element of the matrix $\boldsymbol{A}(n, f), \boldsymbol{a}_{l}^{H}$ denotes the Hermitian transpose of the vector $\boldsymbol{a}_{l}$ and |.| represents the absolute value operator. The measure $\pi_{k l}(n, f)$ takes values between 0 and 1 where high values in a certain T-F bin reflect a directionally linear influence from channel $l$ to channel $k$ in that bin $\left(\mathrm{CH}_{k} \leftarrow \mathrm{CH}_{l}\right)$. Note that the measure is directional, i.e., $\pi_{k l}(n, f)$ is not equal to $\pi_{l k}(n, f)$ necessarily.

\subsection{Time-Varying Orthogonalized PDC}

To reduce the illusive co-variability in power of two signals due to measuring the same sources (the effect of volume conduction), one can orthogonalize them before extracting their power envelopes [15]. Power envelope of a random signal represents the temporal evolution of its spectral power and can be estimated using parametric or non-parametric (FFT-based) methods. It is, however, known that the FFT-based methods inherit performance limitations of the FFT approach. Namely, they are unable to provide high frequency resolution and also, they suffer from the spectral leakage caused by the effect of windowing on the signal. Autoregressive (AR) model-based spectral estimation methods can overcome these limitations by fitting the observations to an AR model. These methods can be extended to multivariate signals using Eq. (1) leading to the power spectral density (PSD) matrix. Therefore, the MVAR model coefficients in Eqs. (1) to (3) reflect the interactions within the channels and at the same time, they represent the spectral information of the signal power envelopes. The main idea behind the orthogonalized PDC (OPDC) measure is that instead of performing the orthogonalization process at the signal level, it can be done at the level of MVAR coefficients. The term $A_{k l}(n, f)$ in Eq. (4) is given by [9]:

$$
A_{k l}(n, f)=\left\{\begin{array}{c}
1-\sum_{r=1}^{p} a_{k l}^{r}(n) e^{-j 2 \pi f r}, \quad \text { if } k=l \\
-\sum_{r=1}^{p} a_{k l}^{r}(n) e^{-j 2 \pi f r}, \text { if } k \neq l .
\end{array}\right.
$$


It turns out that the numerator of the tv-PDC(i.e. $\left.\left|A_{k l}(n, f)\right|\right)$ in Eq. (4) depends directly on the $a_{k l}^{r}(n) e^{-j 2 \pi f r}$ terms which describe the T-F relationships between channel $k$ and channel $l$ at the delay $r$. Each term is a complex number expressed by a real part $a_{k l}^{r}(n) \cos (2 \pi f r)$ and an imaginary part $a_{k l}^{r}(n) \sin (2 \pi f r)$ which are orthogonal in the complex plane:

$$
a_{k l}^{r}(n) e^{-i 2 \pi f r}=a_{k l}^{r}(n) \cos (2 \pi f r)-j a_{k l}^{r}(n) \sin (2 \pi f r) .
$$

The orthogonalized components at different delays don't share the trivial co-variability caused by the effect of mutual sources, as their parallel part has been subtracted. The tv-OPDC is then defined as:

$$
\Psi_{k l}(n, f) \triangleq \frac{\left|\sum_{\mathrm{r}_{1}=1}^{\mathrm{p}} \sum_{\mathrm{r}_{2}=1}^{\mathrm{p}} a_{k l}^{\mathrm{r}_{1}}(n) a_{k l}^{\mathrm{r}_{2}}(n) \cos \left(2 \pi f \mathrm{r}_{1}\right) \sin \left(2 \pi f \mathrm{r}_{2}\right)\right|}{\boldsymbol{a}_{l}^{H}(n, f) \boldsymbol{a}_{l}(n, f)} .
$$

It is straightforward to show that Eq. (7) is equal to:

$$
\Psi_{k l}(n, f)=\frac{\left|\operatorname{Real}\left\{A_{k l}(n, f)\right\}\right|}{\sqrt{\boldsymbol{a}_{l}^{H}(n, f) \boldsymbol{a}_{l}(n, f)}} \cdot \frac{\left|\operatorname{Imag}\left\{A_{k l}(n, f)\right\}\right|}{\sqrt{\boldsymbol{a}_{l}^{H}(n, f) \boldsymbol{a}_{l}(n, f)}}, \quad k \neq l .
$$

Since each factor in Eq. (8) is greater than zero and less than $\pi_{k l}(n, f)$, the measure $\Psi_{k l}(n, f)$ will always take values between zero and 1 .

\subsection{Statistical Testing}

The procedure of obtaining a T-F thresholding plane for each group (left or right hemisphere) of each subject is as follows:

A. tv-OPDC measures are extracted from the whole length (one second) of each epoch. If $N$ is the number of epochs for subject $\boldsymbol{i}$ obtained from either right or left hemisphere, $N$ time-frequency representations of the OPDC measures are obtained at the end of this step.

B. Each time-frequency representation is divided into two parts: first $400 \mathrm{~ms}$ interval and last 400ms interval. First intervals over epochs provide the original estimates and the second intervals build the surrogate library.

C. The highest score at the $99^{\text {th }}$ percentile of the distribution of each T-F bin over epochs is computed. It leads to a thresholding plane covering the whole time-frequency space.

\subsection{Data}

\section{Simulated Data}

Data from a simulated model was used to evaluate the proposed OPDC measure and test the integrity of our time-varying connectivity analysis framework. The model is a 3-dimensional time-varying MVAR(2)-process plus a linear superposition of sparse uniformly distributed random sources, given by: 


$$
\boldsymbol{X}(n)=\boldsymbol{y}(n)+\boldsymbol{V} \boldsymbol{S}(n)
$$

where $\boldsymbol{V} \in \mathbb{R}^{\mathbf{3} \times \mathbf{6}}$ is a time-constant mixing matrix and $\boldsymbol{S}(n)$ denotes the source components matrix. In this study, the elements of $\boldsymbol{V}$ were randomly selected from the interval $[0,1]$. The time-varying MVAR process $\boldsymbol{y}=\left[\begin{array}{lll}y_{1} & y_{2} & \mathrm{y}_{3}\end{array}\right]^{\boldsymbol{T}}$ is expressed as (see also [17]):

$$
\left\{\begin{aligned}
y_{1}(n)= & 0.59 y_{1}(n-1)-0.20 y_{1}(n-2)+ \\
& b(n) y_{2}(n-1)+c(n) y_{3}(n-1)+w_{1}(n) \\
y_{2}(n)= & 1.58 y_{2}(n-1)-0.96 y_{2}(n-2)+w_{2}(n) \\
y_{3}(n)= & 0.60 y_{3}(n-1)-0.91 y_{3}(n-2)+w_{3}(n)
\end{aligned}\right.
$$

where $\boldsymbol{w}=\left[\begin{array}{lll}w_{1} & w_{2} & w_{3}\end{array}\right]^{\boldsymbol{T}}$ is a normally distributed white noise vector. The source components satisfy two assumptions: i) they are independent and ii) they are sparse. The latter property was included in the model, because the EEG source components are known to be sparse in the time-frequency domain [16]. For a model of length $L=2000$ samples and sampling frequency $F_{s}=200 \mathrm{~Hz}$, parameters $b(n)$ and $c(n)$ are a half-sinc and a rectangular function, respectively. For MVAR parameter estimation, the model order is fixed to 2 throughout the process.

\section{Newborn EEG Data}

We used 20-channel EEG data of four healthy full-term newborns obtained from the Department of Children's Clinical Neurophysiology (Helsinki University Central Hospital, Finland). The signals were recorded during sleep with sampling rate of 256 $\mathrm{Hz}$ using a NicOne EEG amplifier (Cardinal Healthcare, USA) and EEG caps (sintered $\mathrm{Ag} / \mathrm{AgCl}$ electrodes; Waveguard, ANT-Neuro, Germany) with positioning according to the international 10-20 standard. To capture connectivity in the whole brain network associated with visual processing (i.e., postcentral) driven by the visual stimuli, we selected ten monopolar channels $\left(\mathrm{C}_{\mathrm{z}}\right.$ as the reference) divided into two groups representing left $\left(\mathrm{O}_{1}, \mathrm{C}_{3}, \mathrm{P}_{3}, \mathrm{~T}_{3}, \mathrm{~T}_{5}\right)$ and right $\left(\mathrm{O}_{2}, \mathrm{C}_{4}, \mathrm{P}_{4}, \mathrm{~T}_{4}, \mathrm{~T}_{6}\right)$ hemispheres. The signals were pre-processed using a $0.1-30 \mathrm{~Hz}$ band-pass filter and ICA for artifact removal. The analysis of functional connectivity was then performed on each hemisphere separately.

\section{Results}

\subsection{Simulation}

Testing the tv-OPDC measure on a simulated process described in Eq. (9) demonstrates that it outperforms the tv-PDC measure in presence of mutual independent sources. Both measures are able to successfully reflect the oscillatory partial connectivity from channel 2 to channel $1\left(\pi_{12}(n, f), \Psi_{12}(n, f)\right)$ as well as the ramp-shaped strength influence from channel 3 to channel $1\left(\pi_{13}(n, f), \Psi_{13}(n, f)\right.$ ) (see Fig. 1). According to the model, there is no direct coupling from $y_{1}(n)$ to $y_{2}(n)$ and $y_{3}(n)$, from $y_{2}(n)$ to $y_{3}(n)$, and also from $y_{3}(n)$ to $y_{2}(n)$. This fact is reflected to the corresponding OPDC plots with negligible T-F values. However, the corresponding PDC plots for $\pi_{21}(n, f), \pi_{23}(n, f), \pi_{31}(n, f)$ and $\pi_{32}(n, f)$ represent high false positive values. Another strong impact can also be observed after applying the proposed approach to the simulated model. The residual connectivity values after removing the effect of mutual sources reveal much smaller magnitude than the 
original PDC values (note the color bars in Fig. 1). This observation originates directly from the orthogonalization step in the OPDC measure where the spurious connectivity caused by the mutual sources is attenuated.
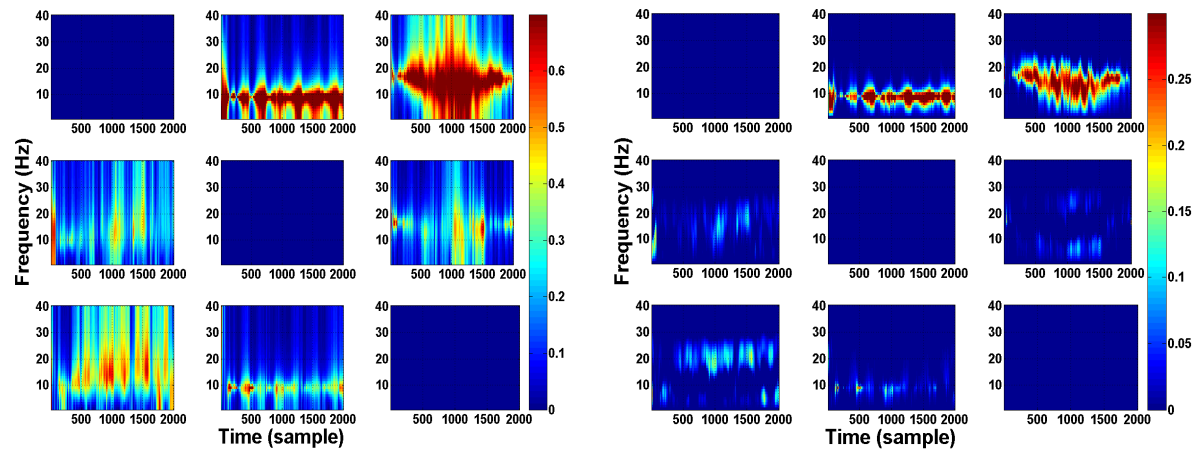

(a)

(b)

Fig. 1. The connectivity measures extracted from the simulated model. a) time-varying PDC, b) time-varying OPDC. The diagonal matrix elements have been set to zero.

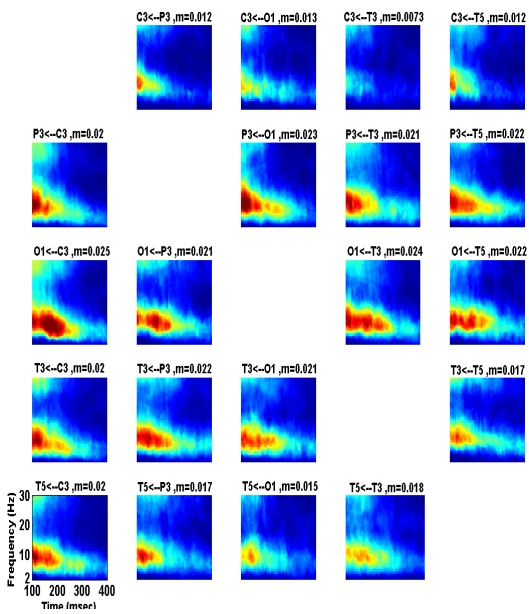

(a)
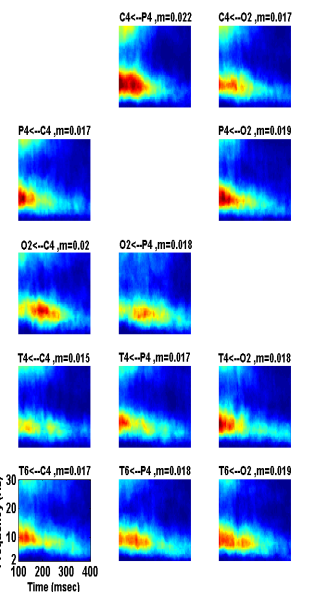

T4< $\cdots 44, \mathrm{~m}=0.015$
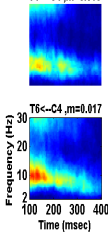

Time (msec)
$P 4<-02, \mathrm{~m}=0.019$
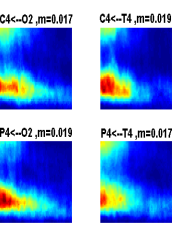

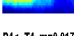

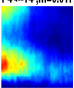

$0<-74 m=002$
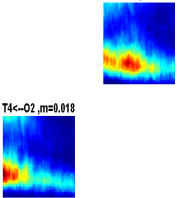

$T \hat{s}<-02, \mathrm{~m}=0.019$

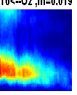

(b)

Fig. 2. Average tv-OPDC measures across four subjects for the occipital-temporal-parietal areas from $100 \mathrm{msec}$ to $400 \mathrm{msec}$ post-stimulus, (a) the left hemisphere, (b) the right hemisphere. The direction of the information flow and the total mean OPDC value [5-15 Hz, 100-300 msec] is presented on top of each map.

\subsection{Time-Frequency Connectivity Analysis on Newborn EEG}

The time-varying connectivity analysis was commenced from $100 \mathrm{~ms}$ post stimulus to cover all cortical components of visual stimuli. Grand mean T-F maps of directional interactions within EEG channels at each hemisphere are demonstrated in Fig. 2. 
An overall inspection of the results in Fig. 2 suggests that there are preferential frequencies and directions of information flow in the T-F domain. To quantify the visual interpretation of the results, the total mean OPDC value is calculated for each plot. The pair-wise OPDC maps, i.e. two maps for each electrode pair (one for each direction) can be interpreted as the pure directional 'coherence spectrogram' between the two electrodes, when the effect of volume conduction is removed. Notably, most directed information flow appears to take place at $5-15 \mathrm{~Hz}$ frequency band, with a general decrease in frequency over time. This change in frequency is, indeed, compatible with the respective changes in the intrinsic frequency content of the average waveform. All graphs show a clear attenuation of interactions towards the end of the 400ms analysis window. The grand mean OPDC maps (Fig. 2) reveal strong interrelations between the occipital and central lobes at the left hemisphere and between the occipital and temporal lobes at the right hemisphere around the central frequency of 10 $\mathrm{Hz}$ (most dominant interactions are $\mathrm{O} 1 \leftarrow \mathrm{C} 3$ and $\mathrm{O} 2 \leftarrow \mathrm{T} 6$ ). In the whole matrices (left and right - 40 maps in total), the dominant electrode pairs involve the occipital electrodes as the sink of information (e.g. $\mathrm{O} 1 \leftarrow \mathrm{T} 3, \mathrm{O} 2 \leftarrow \mathrm{T} 6, \mathrm{O} 1 \leftarrow \mathrm{C} 3$ ). In addition, relatively high $\mathrm{T}-\mathrm{F}$ interactions originate from the temporal lobe and discharge into the

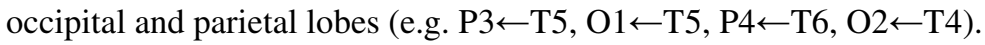

In the next step, we created three-dimensional connectivity maps of the grand mean interactions at $5-15 \mathrm{~Hz}$ band by selecting three time windows $\left(\Delta \mathrm{t}_{1}=100-200 \mathrm{msec}\right.$, $\Delta \mathrm{t}_{2}=200-300 \mathrm{msec}$ and $\Delta \mathrm{t}_{3}=300-400 \mathrm{msec}$ ) depicted in Fig. 3 . The figures verify the observation from the time-frequency OPDC graphs in Fig. 2 that interactions in the network attenuate over time. In the earliest time window (100-200ms), most connections are active, whereas the interactions weaken towards the end of the analysis time. The 3-D maps also imply that long connections travel from the occipital lobe to the central regions during the visual stimuli exposed to the newborn brain.

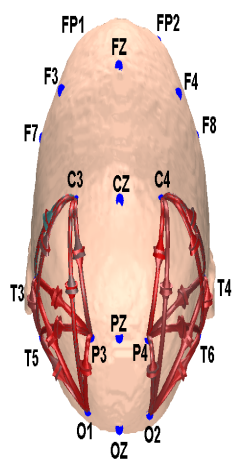

(a)

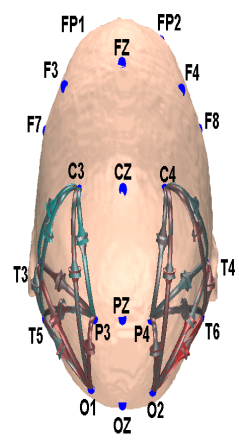

(b)

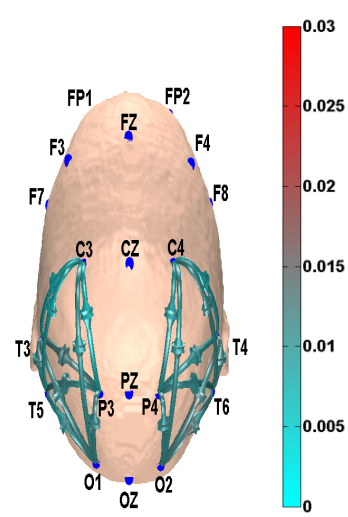

(c)

Fig. 3. Color-coded 3-D directed graphs representing the grand mean information flow at (a) $\Delta \mathrm{t}_{1}=100-200 \mathrm{msec}$, (b) $\Delta \mathrm{t}_{2}=200-300 \mathrm{msec}$ and (d) $\Delta \mathrm{t}_{3}=300-400 \mathrm{msec}$ after the stimulus onset within the frequency range of 5-15 Hz. Note the significant decrease of information flow in the last time window compared to the first two time windows. Each color-coded arrow shows a directed interaction between two electrodes. 


\section{Discussion}

We have demonstrated that tv-OPDC using DEKF is able to track changes associated with transient couplings and remove the effect of mutual independent sources within the multivariate nonstationary signals. Most of the existing EEG connectivity analysis methods assume stationarity of interactions in the underlying signals, while EEG signals are well-known to be nonstationary. Also, the effect of volume conduction is a problematic issue in EEG signal analysis. Our present work introduces a time-frequency framework for functional EEG connectivity analysis to deal with the effect of volume conduction, and extracts the sequence of nonstationary information flows between EEG channels within sub-second segments. Admittedly this is only possible with sufficient signal to noise ratio, which can be achieved by averaging over a larger number of trials as was done in our application.

The effect of EEG montage is an important factor in any studies on EEG connectivity. While we used monopolar montage with $\mathrm{Cz}$ reference in this study, other montages like Laplacian or average referencing should be explored. It would also be useful to explore the potential of the tv-OPDC in source space signals [5] when such become available with the as yet unresolved, realistic inverse solutions for neonatal EEG [17]. Finally, any effect of the number of electrodes in each group on the tv-OPDC measure needs more systematic assessment as volume conduction effects may increase with higher density of EEG electrodes.

The time-varying connectivity measure used in this paper support the same pattern of information transfer in the newborn brain as well. We could observe some longer connections from occipital to temporal and central regions, which is compatible with previous steady state VEP studies in adults $[18,19]$. The proposed methodology in this study from the stimulation paradigm (a routine flash light) and recording system (a routine EEG device) to the analysis procedure with computationally reasonable load make this paradigm directly applicable for large scale clinical trials. Notably, a directed information transfer, called 'travelling waves' in the adult literature [20] is considered to be sensitive to changes in sub-cortical structures [21]. In the clinical context, it raises the potential that our paradigm presented here could be used to assess integrity of the sub-cortical structures after acute brain injury, such as birth asphyxia, where diagnostic strategies have remained a challenge [22]. It may have applicability through change over time in response to therapy and prognostication of long term outcome.

Acknowledgments. This publication was made possible by a grant from the Qatar National Research Fund under its National Priorities Research Program award number NPRP 09-465-2-174. The authors gratefully acknowledge the support of Helsinki University Hospital for providing the newborn EEG datasets. Dr. Sampsa Vanhatalo was supported by the European Community's Seventh Framework Program European Community FP7-PEOPLE-2009-IOF, grant agreement No. 254235.

\section{References}

1. Fisch, B.J.: Fisch \& Spehlmann's EEG primer: Basic principles of digital and analog EEG. Elsevier, Amsterdam (2005)

2. Marinazzo, D., Liao, W., Chen, H., Stramaglia, S.: Nonlinear connectivity by Granger causality. Neuroimage 58, 330-338 (2010) 
3. Milde, T., Leistritz, L., Astolfi, L., Miltner, W.H.R., Weiss, T., Babiloni, F., Witte, H.: A new Kalman filter approach for the estimation of high-dimensional time-variant multivariate AR models and its application in analysis of laser-evoked brain potentials. Neuroimage 50, 960-969 (2010)

4. Lehnertz, K.: Assessing directed interactions from neurophysiological signals-an overview. Physiological Measurement 32, 1715 (2011)

5. Faes, L., Erla, S., Nollo, G.: Measuring Connectivity in Linear Multivariate Processes: Definitions, Interpretation, and Practical Analysis. Computational and Mathematical Methods in Medicine (2012)

6. Cheung, B.L.P., Van Veen, B.D.: Estimation of cortical connectivity from E/MEG using nonlinear state-space models. In: 2011 IEEE International Conference on Acoustics, Speech and Signal Processing (ICASSP), pp. 769-772 (2011)

7. Sommerlade, L., Henschel, K., Wohlmuth, J., Jachan, M., Amtage, F., Hellwig, B., Lücking, C.H., Timmer, J., Schelter, B.: Time-variant estimation of directed influences during Parkinsonian tremor. Journal of Physiology-Paris 103, 348-352 (2009)

8. Geweke, J.: Measurement of Linear Dependence and Feedback Between Multiple Time Series. Journal of the American Statistical Association 77, 304-313 (1982)

9. Baccalá, L.A., Sameshima, K.: Partial directed coherence: a new concept in neural structure determination. Biological Cybernetics 84, 463-474 (2001)

10. Kaminski, M., Blinowska, K.: A new method of the description of the information flow in the brain structures. Biological Cybernetics 65, 203-210 (1991)

11. Korzeniewska, A., Manczak, M., Kaminski, M., Blinowska, K.J., Kasicki, S.: Determination of information flow direction among brain structures by a modified directed transfer function (dDTF) method. Journal of Neuroscience Methods 125, 195-207 (2003)

12. Winterhalder, M., Schelter, B., Hesse, W., Schwab, K., Leistritz, L., Klan, D., Bauer, R., Timmer, J., Witte, H.: Comparison of linear signal processing techniques to infer directed interactions in multivariate neural systems. Signal Processing 85, 2137-2160 (2005)

13. Nolte, G., Bai, O., Wheaton, L., Mari, Z., Vorbach, S., Hallett, M.: Identifying true brain interaction from EEG data using the imaginary part of coherency. Clinical Neurophysiology 115, 2292-2307 (2004)

14. Gomez-Herrero, G.: Brain Connectivity Analysis with EEG. Doctoral dissertation, Department of Signal Processing, Tampere University of Technology, Finland (2010)

15. Hipp, J.F., Hawellek, D.J., Corbetta, M., Siegel, M., Engel, A.K.: Large-scale cortical correlation structure of spontaneous oscillatory activity. Nat. Neurosci. 15, 884-890 (2012)

16. Yuanqing, L., Cichocki, A., Amari, S.I.: Blind estimation of channel parameters and source components for EEG signals: a sparse factorization approach. IEEE Transactions on Neural Networks 17, 419-431 (2006)

17. Roche-Labarbe, N., Aarabi, A., Kongolo, G., Gondry-Jouet, C., Dumpelmann, M., Grebe, R., Wallois, F.: High-resolution Electroencephalography and source localization in neonates. Human Brain Mapping 29, 167-176 (2008)

18. Yan, Z., Gao, X.: Functional connectivity analysis of steady-state visual evoked potentials. Neuroscience Letters 499, 199-203 (2011)

19. Srinivasan, R., Fornari, E., Knyazeva, M., Meuli, R., Maeder, P.: fMRI responses in medial frontal cortex that depend on the temporal frequency of visual input. Experimental Brain Research 180, 677-691 (2007)

20. Burkitt, G.R., Silberstein, R.B., Cadusch, P.J., Wood, A.W.: Steady-state visual evoked potentials and travelling waves. Clinical Neurophysiology 111, 246-258 (2000)

21. Nunez, P.L.: Implications of white matter correlates of EEG standing and traveling waves. Neuroimage 57, 1293-1299 (2010)

22. Thoresen, M., Hellström-Westas, L., Liu, X., de Vries, L.S.: Effect of Hypothermia on Amplitude-Integrated Electroencephalogram in Infants With Asphyxial. Pediatrics 126, $131-139$ (2010) 